\title{
Study on Highway Skid Resistance Evaluation with Safety Factors
}

\author{
Rihua Bai, Lijian Mao, Zhanshun Bo, Rihua Bai, Lijian Mao and Zhanshun Bo \\ Research Institute of Highway Ministry of Transport
}

\begin{abstract}
Key words: maintenance quality evaluation; skid resistance evaluation; safety factors; skid resistance require-ments.

Abstract: Pavement skid resistance condition should be taken into account with due care when evaluating the highway maintenance qualities, because it is related to the users' life and property. The research proposes skid resistance evaluation methods (safety focused) and the main evaluation parameters on the basis of current 'Highway Performance Assessment Standards', domestic and overseas research results, and the practical works. The author believes that the results of this research will be used as references and contribute to the improvement of current standards.
\end{abstract}

\section{Introduction}

In recent years, the general scale of China's transportation infrastructure constructions has been increased rapidly, as a result it gradually entering itself into a large scale of highway maintenance age. Highway maintenance is a long-term, on going and periodic process, it needs a real time tracking and monitoring the maintenance quality of highway in order to develop an appropriate evaluation. In practice, the author found that current evaluation standards do not pay a sufficient attention on safety factors in skid resistance evaluation

\section{Lower Level Highway Evalution should include the Skid Resistance Factor}

According to the current standards, only the skid resistance performance of highway and arterial road is required to be examined on a biennial basis. This is because such road refers to channelization. It has features of high speed and high volume. Therefore, the evaluation of skid resistance is necessary for safety issuers. However, lower level highways still contains huge potential safety risks if they have a poor skid resistance performance. The serious traffic accident below demonstrates the necessity of the requirement of skid resistance performance for low speed roads.

In October 13, 1988, a light bus with 18 passengers lost control and slide out the road when passing Line ZQ K34+ 014. The investigation report shows 12 people died in the accident. The cause has been identified as the poor skid resistance performance of the road. The road was under construction of paving the new layer, every performance indicator had been met as the standards required. However, with the absence of any standards regarding the skid resistance performance for such road, the accident paid the price.

Although it is a extreme events, it sill raise the implications on the mportance of skid resistance performance in pavement maintenance quality evaluations, and such performance should be considered as the minimal requirement regarding to the safety issues.

In addition, some related regulations, such as 'Standards For Highway Engineering Quality Evaluation'; 'Technical Specification For Maintenance Of Asphalt Pavement' and 'Technical Specification For Maintenance Of Cement Concrete Pavement', do require skid resistance performance for lower level road. Therefore, the author believes it is necessary to take the skid resistance factor into account when evaluating the lower level road conditions from either the safety aspect or regulation consistency aspect. 


\section{The Skid Resistance Standards should be based on the Requirements of the Road}

The current standards for highway maintenance quality are demonstrated by the MQI and corresponding sub indicators. The range is $0-100$. The maintenance qualities can be categorized into five different levels: excellent, good, fair, poor, and very poor. The maintenance quality level then can be determined by standards in table 1 :

Table 1. Standards For Highway Maintenance Quality Evaluation.

\begin{tabular}{cc}
\hline Level & MQI and corresponding sub indicators \\
\hline Excellent & $\geq 90$ \\
Good & $\geq 80,<90$ \\
Fair & $\geq 70,<80$ \\
Poor & $\geq 60,<70$ \\
Very poor & $<60$ \\
\hline
\end{tabular}

Skid resistance performance is measured by Skid Resistance Indicator (SRI); the equation is illustrated as follows:

$$
S R I=\frac{100-S R I_{\min }}{1+\alpha_{0} e^{\alpha_{1} S F C}}+S R I_{\text {min }}
$$

Where: $S F C$ is transverse force

$S R I_{\min }$ is calibration coefficient

$\alpha_{0}$ and $\alpha_{1}$ are the model parameter

Note: the calibration coefficient and the model parameter are constant value in the equation.

As can be seen the equation of current standard only allows one variable, i.e. SFC. It is independent from other indicators, weather conditions and traffic factors.

If the pavement SRI is under the required minimum value, the drivers are more likely to loose control especially on the curve, descent and roundabout.

The regulations in foreign countries are generally considering the specific conditions of the road. For instance, the pavement friction performance is measured by SFC in the UK, and it also provides different SFC for different road sections that will be illustrated in Table 2.

Table 2. Requirements on pavement SFCs in the UK.

\begin{tabular}{|c|c|c|}
\hline Level & Road section & SFC \\
\hline A、B & super highway & 45 \\
\hline C、D & secondary junctions & 51 \\
\hline E、F & entrance and exit of primary junctions & 58 \\
\hline G1 & longitudinal gradient $5 \% \sim 10 \%$ & 58 \\
\hline $\mathrm{G} 2$ & longitudinal gradient $\geq 10 \%$ & 64 \\
\hline $\mathrm{H}$ & $\mathrm{R} \leq 250 \mathrm{~m}, \quad \mathrm{~V} \leq 64 \mathrm{~km} / \mathrm{h}$ & 58 \\
\hline I、J & $\mathrm{R} \leq 100 \mathrm{~m}, \quad \mathrm{~V} \leq 64 \mathrm{~km} / \mathrm{h}$ & 64 \\
\hline $\mathrm{K}$ & near the traffic signals & 64 \\
\hline $\mathrm{L}$ & rotary intersection & 58 \\
\hline
\end{tabular}

In addition, when the pavements are wet, no matter how much attention has been paid by the driver, the danger of sideslipping still exists. A great number of researches show that wet traffic accidents are well related to the road skid resistance performance. If the traffic and rainfall conditions are the same, the wet traffic accident rate is negatively correlated to the SFC.

Therefore, the author argues that it might not be appropriate to adopt the unified skid resistance requirements by not taking different road conditions into account. 


\section{Setting sri threshold}

In current standards ('Standards for Highway Maintenance Quality Evaluation'), the evaluation covers four different areas: pavement, subgrade, Bridge, and traffic-facility. Evaluation indicators are in Table 3; the range for all indicators is $0-100$.

MQI is calculated by equation 2 :

$M Q I=\omega_{P Q I} P Q I+\omega_{S C I} S C I+\omega_{B C I} B C I+\omega_{T C I} T C I$

Where $\omega_{P Q I}, \omega_{S C I}, \omega_{B C I}, \omega_{T C I}$ are the corresponding weightings of sub-indicators.

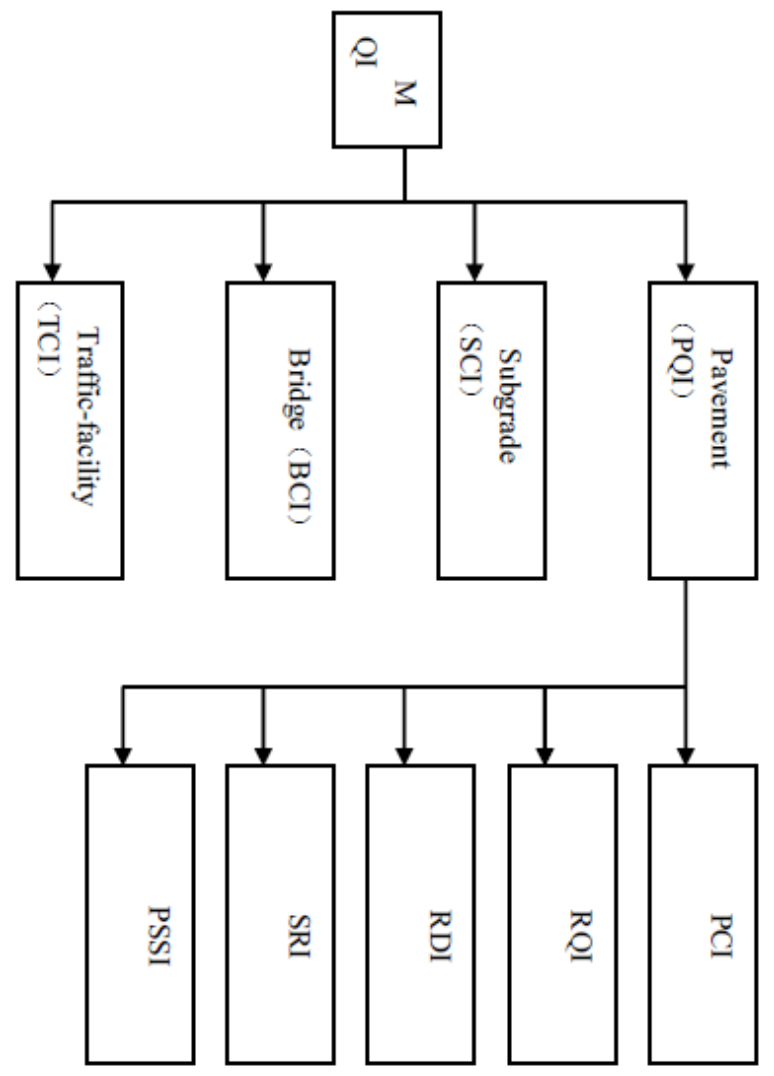

Table 3. Maintenance Quality Indicators

In regards to the road section contains the five kinds of bridge, dangerous tunnel and dangerous culvert, the MQI equals to 0.

$\mathrm{PQI}$ in the above equation is calculated as follows:

$$
P Q I=\omega_{P C I} P C I+\omega_{R Q I} R Q I+\omega_{R D I} R D I+\omega_{S R I} S R I
$$

Where $\omega_{P C I}, \omega_{R Q I}, \omega_{R D I}, \omega_{S R I}$ are the corresponding weightings of sub-indicators.

According to the above equations, SRI has a relatively low weighting. Therefore, even if the SRI equals to 0 , it still has a low impact on the MQI evaluation.

Once the pavement skid resistance performance is under certain levels, it will bring serious threats to the drivers. Therefore, there should be a threshold value for SRI. When road SRI is below the threshold, the MQI of such road equals to 0 .

\section{Multi indicators skid resistance evaluation (safety focused)}

Research shows, the anti-skidding performance of pavement is primarily affected by vehicles, road, and environment. It is very rare that Traffic safety issues are caused by a single element; normally, 
they are determined by the combined action of several different elements. Therefore, it is necessary to establish a Multi indicators skid resistance evaluation system (safety focused). When the section and traffic condition are fixed, road condition and the environment become to the primary determinant of anti-skidding performance, thus the skid resistance evaluation system should introduce road-related and natural condition-related parameters. Road condition is comprised of natural conditions and practical conditions. As the basic elements of road traffic, technical grade, facilities condition, traffic environment and other natural conditions are fundamentally important to traffic safety. Therefore, the natural condition-related parameters might include radii of horizontal, longitudinal slope, the ultra-high and high breaking requirements and etc. On the other hand, among the practical condition-related parameters, the friction coefficient is the most significant parameter to traffic safety, as it has a direct influence on emergency breaking distance and lateral stability. In addition, the pavement friction coefficient will be further reduced by special weathers such as fog spray, rain and snow spray, and icy pavement. As to the practical condition-related parameters, the lateral force coefficient is the most important one that need to be taken into account. Environmentrelated parameters might include precipitation and the temperature. It is the common sense that the number of accident in rainy days is greater than in normal weathers. Therefore, the current 'Specifications for Design of Highway Asphalt Pavement' should take precipitation into account and adopt different skid resistance standards at the design phase. Different seasons also give rise to different pavement skid resistance indicators, there are researches shows that the order of pavement skid resistance performance of the same road section in different seasons is as follows: winter > spring and autumn $>$ summer.

\section{Conclusions}

As a very important indicator with regard to users' life and property issues, pavement skid resistance performance should be seriously considered maintenance quality evaluations. By discussing the current standards combined with practical works, the author made several recommendations. Hopefully these recommendations will be used as references and contribute to the improvement of current standards.

\section{References}

[1] Highway Performance Assessment Standards. Beijing: China Communications Press, 2008,In Chinese.

[2] Quality Inspection and Evaluation Standards for Highway Engineering: China Communications Press, 2004,In Chinese.

[3] Technical for Maintenance of Highway Asphalt Pavement: China Communications Press, 2001,In Chinese.

[4] Technical Specifications of Cement Concrete Pavement Maintenance for Highway: China Communications Press, 2001,In Chinese.

[5] Zou xi , Zhu xiaobo , Ding Tongqiang:Journal of Jilin University of Technology(Natural Science Edition) ,2001,31(3):53-56,In Chinese.

[6] Liu mingzhi: Research of Environmental Factors on The Asphalt Pavement Anti-sliding Performance Impact: Harbin Institute of Technology, 2016, In Chinese.

[7] Zhu yunsheng, Xiang huilun, Zhang xiedong, Zhang yan: Journal of Wuhan University of Technology (Transportation Science \& Engineering),2012,36(1):6-10,In Chinese. 